\title{
Extremum Seeking Algorithms for Minimal Hydrogen Consumption in PEM Fuel Cells*
}

\author{
Cristian Kunusch $^{1} \quad$ Fernando Castaños ${ }^{2}$
}

\begin{abstract}
We present an adaptive sliding-mode extremum seeker that minimizes an unknown function that is subject to an unknown static constraint. The same algorithm can be applied when the static constraint is replaced by a dynamic one, provided that the dynamics possess strong stability properties. The application and feasibility study is focused on hydrogen consumption minimization in PEM fuel cell based systems.
\end{abstract}

\section{INTRODUCTION}

Increasing demands on pollution reduction is driving innovation on clean energy sources. Among these, fuel cells (FCs) are regarded as one of the most promising technologies, due to their potential efficiency, compactness and reliability [2]. FCs are electrochemical devices that generate electrical energy from hydrogen and oxygen, with pure water and heat as by-products. Considering that hydrogen is widely available and can be obtained from many renewable sources using solar and wind energy, fuel cells represent an attractive, feasible alternative to reduce fossil fuel dependence. However, the widespread use of hydrogen as combustible -and the resulting "hydrogen economy"- despite its interesting possibilities, has some technological issues to be resolved. In spite of recent advances, their relatively high costs, improvable efficiency and reduced lifetime remain as major limitations. For this reason, together with the continuous improvement of materials and components, the incorporation of advanced control strategies embodies a major technological issue, in order to achieve cost reduction, performance improvement and efficiency optimization. In the light of these considerations, it becomes clear that in order to optimize efficiency, hydrogen minimization problem arises as a major challenge. Therefore, the current paper addresses the analysis and design of optimizing supervisory controllers for fuel cell systems. In particular, robust extremum seeking algorithms based on the so called sliding-mode paradigm are considered for the case of air flow reference management vs. stack current minimization.

Extremum seeking algorithms deal with the problem of minimizing or maximizing a plant output with a set of decision variables. This problem represents a class of

*This research has been supported by the Seventh Framework Programme of the European Community through the Marie Curie actions (GA: PCIG09GA-2011-293876), Puma-Mind project (GA: FCH-JU-2011-1-303419), the CICYT project DPI2011-25649 (MICINN-España), the CSIC JAE-DOC Research Programme.

${ }^{1}$ C. Kunusch is with Institut de Robòtica i Informàtica Industrial (CSICUPC), Barcelona, Spain. ckunusch@iri.upc.edu

${ }^{2}$ F. Castaños is with Centro de Investigación y de Estudios Avanzados del Instituto Politécnico Nacional (CINVESTAV), Mexico D.F., Mexico. castanos@ieee.org widespread optimization problems arising in different control applications.

In the proposed approach, a general extremum seeking algoritm is first devised for a static plant. This is done by estimating the gradient of the output and then applying a standard steepest descent algorithm. The input and output of the plant are differentiated, and then the gradient of the plant is extracted with a sliding-mode adaptive estimator. We claim that the algorithm also works for dynamic plants if the plant satisfies some stability properties. The claim is supported by simulating the behavior of the extremum seeker when applied to an FC.

The system under evaluation is composed of an autonomous PEM fuel cell generation system (FCGS). The FCGS roughly comprises five main subsystems: the air flow (breathing), hydrogen flow, gases humidity, stack electro chemistry and stack temperature subsystems. It is assumed that the input reactant flows are efficiently humidified and the stack temperature is well regulated by dedicated controllers. In addition, it is considered that sufficient compressed hydrogen is available, therefore the main attention is focused on the air management. In Fig. 1 a schematic view of the FCGS under consideration is represented. The most relevant components related to the FC flow system are deeply characterized in [6].

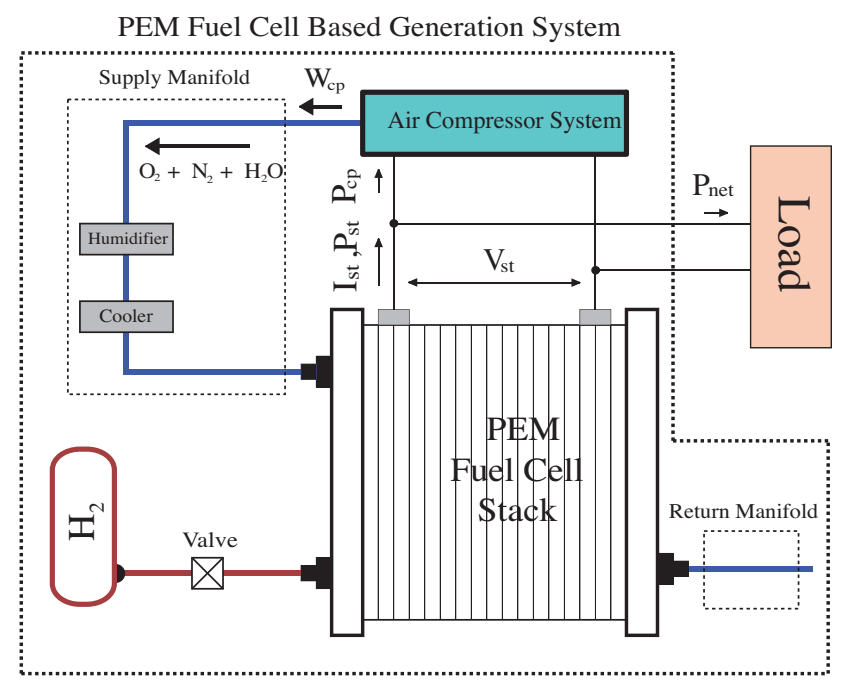

Fig. 1. Schematic diagram of a typical PEM fuel cell based generation system (FCGS) 


\section{AdAptive Extremum SeEking}

\section{A. Static case}

Consider the problem of minimizing a smooth real-valued objective function

$$
y=h(x, u), \quad x \in \mathbb{R}^{n}, u \in \mathbb{R},
$$

subject to the constraint $0=f(x, u)$ and let us state the following assumptions.

Assumption 1: There exists a smooth function $\phi: \mathbb{R} \rightarrow \mathbb{R}^{n}$ such that

$$
0=f(x, u) \quad \text { if and only if } \quad x=\phi(u) .
$$

Assumption 2: The function $H(u):=h(\phi(u), u)$ is twice continuously differentiable and there is an open interval $\mathscr{D}$ of interest, such that:

1) The inequalities

$$
\rho_{1} \leq \frac{\partial^{2} H}{\partial u^{2}}(u) \leq \rho_{2}
$$

hold for some $\rho_{1}, \rho_{2}>0$ and all $u \in \mathscr{D}$. (Hence, $H$ is strictly convex when restricted to $\mathscr{D}$.)

2) The set

$$
\mathscr{Z}:=\left(\frac{\partial H}{\partial u}\right)(\mathscr{D})=\left\{z \in \mathbb{R}: z=\frac{\partial H}{\partial u}(u), u \in \mathscr{D}\right\}
$$

contains the origin.

If $\phi$ and $h$ were known, then the optimal pair

$$
\left(x^{\star}, u^{\star}\right)=\underset{0=f(x, u), u \in D}{\arg \min } h(x, u)
$$

could be easily found by solving

$$
0=\frac{\partial H}{\partial u}\left(u^{\star}\right)=\left(\frac{\partial h}{\partial x} \frac{\partial \phi}{\partial u}+\frac{\partial h}{\partial u}\right)\left(u^{\star}\right)
$$

(such a solution always exists under condition (2)) and setting $x^{\star}=\phi\left(u^{\star}\right)$. On the other hand, the optimization problem becomes more challenging and of greater practical interest when $y$ is available (e.g., from measurements) but $\phi$ and $h$ are not known. To solve this problem, we suggest to estimate $\frac{\partial H}{\partial u}$ and feed it to a continuous-time steepest descent algorithm

$$
\dot{u}(t)=-\mathbf{1}_{\left[t_{1}, \infty\right)}(t) \cdot k_{u} z(t)+d(t),
$$

where $z(t) \in \mathbb{R}$ is an estimate of $\frac{\partial H}{\partial u}$ at time $t, k_{u} \in \mathbb{R}$ is the algorithm gain and $\mathbf{1}_{\left[t_{1}, \infty\right)}: \mathbb{R} \rightarrow\{0,1\}$ is the indicator function of the interval $\left[t_{1}, \infty\right)$, i.e., we 'turn' $k_{u} z(t)$ 'on' only when $t \geq t_{1}$. The switching time is to be defined later (see (12)). The term $d(t)$ is a differentiable signal included to ensure a persistent excitation for the estimator. It is chosen in such a way that it satisfies the bounds

$$
|d(t)| \leq \bar{d}_{0} \quad \text { and } \quad|\dot{d}(t)| \leq \bar{d}_{1},
$$

where $\bar{d}_{0}$ and $\bar{d}_{1}$ are positive constants to be determined later.

We propose an adaptive sliding-mode estimator of the form

$$
\dot{z}(t)=k_{z} \operatorname{sign}((\dot{y}-z \dot{u}) \dot{u})(t), \quad z(0)=0 .
$$

where $k_{z} \in \mathbb{R}$ is the estimator gain and $\dot{y}$ is obtained using the uniform exact differentiator described in Section II-C.

Lemma 1: Suppose that $\dot{u}(t) \neq 0$ almost everywhere (a.e.) (this is our persistence of excitation condition), let $k_{z}$ be such that

$$
k_{z} \geq \rho_{2}\left(k_{u}|z|+\bar{d}\right)+\delta_{0}, \quad z \in \mathscr{Z}
$$

for some $\delta_{0}>0$ and suppose that $\frac{\partial H}{\partial u}(t) \in \mathscr{Z}$ for $t \geq 0$. Then, the solutions $z(t)$ of the estimator (5) converge to $\frac{\partial H}{\partial u}(t)$ in finite time.

Proof: Notice that

$$
\dot{y}(t)=\frac{\partial h}{\partial x} \dot{x}(t)+\frac{\partial h}{\partial u} \dot{u}(t)
$$

and $\dot{x}(t)=\frac{\partial \phi}{\partial u} \dot{u}(t)$, so we have

$$
\dot{y}(t)=\left(\frac{\partial h}{\partial x} \frac{\partial \phi}{\partial u}+\frac{\partial h}{\partial u}\right) \dot{u}(t)=\frac{\partial H}{\partial u} \dot{u}(t) .
$$

Using (7) we can rewrite (5) as $\dot{z}(t)=$ $k_{z} \operatorname{sign}\left(\left(\frac{\partial H}{\partial u}-z\right) \dot{u}^{2}\right)(t)$. Since $\dot{u}(t) \neq 0$ almost everywhere, we have

$$
\dot{z}(t)=k_{z} \operatorname{sign}\left(\frac{\partial H}{\partial u}-z\right)(t) \text { a.e. }
$$

Let $s:=\frac{\partial H}{\partial u}-z$ be the sliding variable. Its time derivative is given by

$$
\dot{s}(t)=\frac{\mathrm{d}}{\mathrm{d} t} \frac{\partial H}{\partial u}(t)-\dot{z}(t)=-k_{z} \operatorname{sign}(s(t))+\frac{\mathrm{d}}{\mathrm{d} t} \frac{\partial H}{\partial u}(t) \text { a.e. }
$$

Now we can take the standard approach to prove that $s(t) \rightarrow 0$ in finite time: Define a Lyapunov function $V_{s}(s)=s^{2} / 2$ and compute its time derivative along the trajectories of (9), i.e.,

$$
\begin{aligned}
\dot{V}_{s}(t)=s \cdot \dot{s}(t) & \leq-|s|\left(k_{z}-\left|\frac{\mathrm{d}}{\mathrm{d} t} \frac{\partial H}{\partial u}\right|\right)(t) \leq \\
& \leq-|s|\left(k_{z}-\rho_{2}|\dot{u}|\right)(t) \text { a.e. }
\end{aligned}
$$

(with a slight abuse of notation, we have set $V_{s}(t)=V_{s}(s) \circ$ $s(t)$ ). From $|\dot{u}| \leq k_{u}|z|+\bar{d}$ (cf. (4)), (6) and (10), we have

$$
\dot{V}_{s}(t) \leq-\delta_{0}|s(t)| \text { a.e. }
$$

Thus, the time derivative is negative. This proves that the point $s=0$ is an asymptotically stable equilibrium of (9). To show convergence in finite time, notice that (10) can be rewritten as

$$
\dot{V}_{s}(t) \leq-\delta_{0} \sqrt{V_{s}(t)} \text { a.e., }
$$

which implies that [9]

$$
V_{s}(t) \leq\left(\sqrt{V_{s}(0)}-\frac{\delta_{0}}{2} t\right)^{2}
$$

It follows that at the time

$$
t_{1}=2 \frac{\sqrt{V_{s}(s(0))}}{\delta_{0}}
$$

we have $V_{s}\left(t_{1}\right)=0$ (hence $s\left(t_{1}\right)=0$ ). 


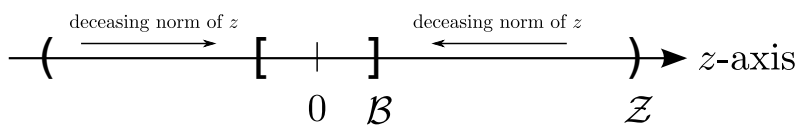

Fig. 2. Proof of Theorem 1

Remark 1: Instead of (12) (which requires knowledge of $s(0)$ ), the switching time $t_{1}$ can be determined online by monitoring the error $e:=\dot{y}-z \dot{u}$ and marking the time where $e=0$ with $\dot{u} \neq 0$.

Because of the presence of $d(t), z=\frac{\partial H}{\partial u}$ cannot be made exactly equal to zero, but it can be driven to a small value proportional to $\bar{d}$.

Theorem 1: Let $\mathscr{B}:=\left[-\bar{d} / k_{u}, \bar{d} / k_{u}\right]$ and suppose that:

1) $\mathscr{B} \subset \mathscr{Z}$.

2) $\dot{u}(t) \neq 0$ a.e. and $k_{z}(z)$ satisfies (6).

3) $z\left(t_{1}\right) \in \mathscr{Z}$.

Then, it follows from equations (5) and (4) that $\mathscr{B}$ and $\mathscr{Z}$ are positive invariant sets and that $z(t) \rightarrow \mathscr{B}$ as $t \rightarrow \infty$.

Proof: Define a candidate Lyapunov function $V_{z}(z)=$ $z^{2} / 2$. From Lemma 1 we know that, during the sliding motion (i.e., for $\left.t \geq t_{1}\right), z(t)=\frac{\partial H}{\partial u}(t) \mathrm{so}^{1}$

$$
\dot{z}(t)=\frac{\mathrm{d}}{\mathrm{d} t} \frac{\partial H}{\partial u}(t)=\frac{\partial^{2} H}{\partial u^{2}} \dot{u}(t)=-\frac{\partial^{2} H}{\partial u^{2}}\left(k_{u} z(t)-d(t)\right),
$$

and

$\dot{V}_{z}(t)=-\frac{\partial^{2} H}{\partial u^{2}} \cdot\left(k_{u} z^{2}-z \cdot d\right)(t) \leq-\frac{\partial^{2} H}{\partial u^{2}}|z| \cdot\left(k_{u}|z|-\bar{d}\right)(t)$.

The terms $\frac{\partial^{2} H}{\partial u^{2}}$ and $k_{u}|z|-\bar{d}$ are positive whenever $z \in \mathscr{Z}$ and $z \notin \mathscr{B}$, respectively. Thus, $\dot{V}_{z}$ is negative whenever $z \in \mathscr{Z}-\mathscr{B}$ and $|z|$ decreases monotonically whenever $z \in \mathscr{Z}-\mathscr{B}$. This implies that $\mathscr{B}$ and $\mathscr{Z}$ are positive invariant sets and that $z(t) \rightarrow \mathscr{B}$ as $t \rightarrow \infty$ whenever $z\left(t_{1}\right) \in \mathscr{Z}$ (see Fig. 2).

Theorem 1 suggests the following practical considerations for designing the extremum seeker (4), (5):

1) Choose a gain $k_{u}$ for the steepest-descent algorithm, a constant $\delta_{0}>0$ and a strict subinterval $\mathscr{I}$ of $\mathscr{D}$ of initial conditions for $u$.

2) For $d(t)$, use a periodic signal with zero average and amplitude $\bar{d}$. Choose $\bar{d}$ small enough so that (a)

a) $\mathscr{B} \subset \mathscr{Z}$

b) $u(0) \in \mathscr{I}$ implies $u(t) \in \mathscr{D}$ for all $t \in\left[0, t_{1}\right]$ (this ensures that $\left.z\left(t_{1}\right) \in \mathscr{Z}\right)$.

3) Set the estimator gain as $k_{z}(z)=k_{u}|z|+\bar{d}+\delta_{0}$.

\section{B. Dynamic case}

To alleviate the notation, we will now drop $t$ from the functions' arguments whenever the dependence is obvious.

Consider now the problem of minimizing the output (1) that results from the dynamic relation

$$
\dot{x}=f(x, u)
$$

\footnotetext{
${ }^{1}$ This equation could also be obtained by applying the equivalent control method [8] to (8).
}

Suppose we are interested in driving the state $x$ to the optimal equilibrium pair (2). If $\frac{\partial H}{\partial u}$ was known and the equilibrium $\left(\phi\left(u^{\star}\right), u^{\star}\right)$ is at least asymptotically stable, then the optimal value could be reached by setting

$$
\dot{u}=g(u):=-k_{u} \frac{\partial H}{\partial u}(u),
$$

so extremum seeking problem reduces again to the problem of estimating $\frac{\partial H}{\partial u}$ online.

If the solutions $x$ of (13) converge to $\phi(u)$ fast enough and $\dot{x}$ quickly comes close to $\frac{\partial \phi}{\partial u} \dot{u}$ as well, then it makes sense to apply again the algorithm (4), (5), which we now know works for the case $x=\phi(u)$.

To motivate our next assumption, consider a linear system

$$
\dot{x}=A x+B u
$$

with $A$ Hurwitz. For each constant $u$, the equilibrium is given by $x=\phi(u)=-A^{-1} B u$. Let us define the error

$$
e=x-\phi(u)
$$

an let us compute its dynamics:

$$
\dot{e}=A(e+\phi(u))+B u-\frac{\mathrm{d}}{\mathrm{d} t} \phi(u)=A e+A^{-1} B \dot{u} .
$$

We know from linear system theory that the error satisfies the bound (see, e.g., [10, Sec. 4.9])

$$
\|e(t)\| \leq \alpha \exp (-\lambda t)\|e(0)\|+\frac{\alpha}{\lambda}\|B\| \sup _{0 \leq \tau \leq t}|\dot{u}(\tau)|
$$

for some positive constants $\alpha$ and $\lambda$. A similar bound can be found for $\dot{e}$ if we compute the second derivative of the error. We have

$$
\ddot{e}=A \dot{e}-A^{-1} B \ddot{u}
$$

and

$$
\|\dot{e}(t)\| \leq \alpha \exp (-\lambda t)\|\dot{e}(0)\|+\frac{\alpha}{\lambda}\|B\| \sup _{0 \leq \tau \leq t}|\ddot{u}(\tau)| .
$$

This estimate shows that the zero-input (taking $\ddot{u}$ as input) response decays to zero exponentially fast, while the zerostate response remains bounded for every bounded input. In other words, the dynamics for the error is input-state stable.

In the general nonlinear case, the error equation is

$$
\dot{e}=f(e+\phi(u), u)-\frac{\partial \phi}{\partial u} \dot{u} .
$$

Notice that, for $\dot{u} \equiv 0$ the origin $e=0$ is an equilibrium of (15), uniformly in $u$.

Assumption 3: There are exists positive constants $\alpha$ and $\lambda$ and a class $\mathscr{K}$ function $\gamma$ such that

$$
\|\dot{e}(t)\| \leq \alpha \exp (-\lambda t)\|\dot{e}(0)\|+\gamma\left(\sup _{0 \leq \tau \leq t}|\ddot{u}(\tau)|\right)
$$

for all $t \geq 0$, uniformly in $u$ and $\dot{u}$.

Remark 2: It follows form (4) and (5) that

$$
|\ddot{u}| \leq k_{u} k_{z}+\bar{d}_{1} .
$$

so the time derivative of the error will decay, exponentially fast, to a small neighborhood of zero if $k_{u}$ and $\bar{d}_{1}$ are small. 
Let us write the output's time derivative as

$$
\dot{y}=\frac{\partial h}{\partial x}\left(\dot{e}+\frac{\partial \phi}{\partial u} \dot{u}\right)+\frac{\partial h}{\partial u} \dot{u}=\frac{\partial H}{\partial u} \dot{u}+\frac{\partial h}{\partial x} \dot{e}
$$

(cf. equation (7)). The estimator (5) can thus be written as

$$
\dot{z}=k_{z} \operatorname{sign}\left(\left(\frac{\partial H}{\partial u}-z\right) \dot{u}^{2}+\frac{\partial h}{\partial x} \dot{e} \dot{u}\right) .
$$

Under the persistence of excitation condition we can write

$$
\dot{z}=k_{z} \operatorname{sign}\left(\frac{\partial H}{\partial u}-z+\frac{\partial h}{\partial x} \frac{\dot{e}}{\dot{u}}\right) \quad \text { a.e. }
$$

or, more compactly,

$\dot{z}=k_{z} \operatorname{sign}(s), \quad s=f_{1}-z+f_{2}, \quad f_{1}:=\frac{\partial H}{\partial u}, \quad f_{2}:=\frac{\partial h}{\partial x} \frac{\dot{e}}{\dot{u}}$.

At the sliding surface, $z$ is equal to $\frac{\partial H}{\partial u}$ plus an error $f_{2}$, proportional to $k_{u} k_{z}+\bar{d}_{1}$ (cf. Remark 2) and inversely proportional to $\dot{u}$. Since $f_{2}$ can grow without bound as $\dot{u}$ gets close to zero, it is clear that it is not possible (nor desirable) to maintain the sliding motion all times. Thus, we proceed with a 'heuristic' analysis of what happens when the sliding motion occurs and when the sliding motion is lost.

By repeating the Lyapunov analysis of Section II-A, we can see that the estimator drives $z$ to the sliding surface whenever

$$
k_{z}>\left|\dot{f}_{1}\right|+\left|\dot{f}_{2}\right| .
$$

The time derivative of $f_{1}$ is not problematic and has been address in (6). Let us write

$$
f_{2}=\frac{f_{3}}{\dot{u}}, \quad f_{3}:=\frac{\partial h}{\partial x} \dot{e},
$$

so that we can write $\dot{f}_{2}$ as

$$
\dot{f}_{2}=\frac{\dot{f}_{3} \dot{u}-\ddot{u} f_{3}}{\dot{u}^{2}} \text {. }
$$

From the sliding condition (16) and the expressions for $f_{2}$ and $\dot{f}_{2}$ we arrive at the following two possibilities:

1) $\dot{u}$ is large enough. Then the sliding condition holds and $z$ tracks $\frac{\partial H}{\partial u}+f_{2}$ with $f_{2}$ small.

2) $\dot{u}$ is too small. Then the sliding condition does not hold and $z$ does not track $\frac{\partial H}{\partial u}+f_{2}$ with $f_{2}$ large.

In the numerical experiments performed so far, by setting $k_{z}$ high enough, the tracking error $z-\frac{\partial H}{\partial u}$ can be made reasonably small.

\section{Uniform Exact Differentiator}

The time functions $\dot{u}$ and $\dot{y}$ can be computed with a uniform exact differentiator [1], a differentiator based on the generalized super twisting algorithm [7].

Let $\eta(t)$ be a Lebesgue-measurable function defined on $[0, \infty)$ and take it as the input signal. Suppose that $\eta(t)$ can e decomposed as

$$
\eta(t)=\eta_{0}(t)+v(t)
$$

where $\eta_{0}(t)$ is the unknown base signal that we wish to differentiate and $v(t)$ corresponds to a uniformly bounded noise signal. We assume that $\eta_{0}(t)$ is twice continuously differentiable and that the first derivative is Lipschitz with known Lipschitz constant $L>0$.

Define the error signal $\sigma=z_{0}-\eta$ and construct the dynamical system

$$
\begin{aligned}
& \dot{z}_{0}=-k_{1} \phi_{1}(\sigma)+z_{1} \\
& \dot{z}_{1}=-k_{2} \phi_{2}(\sigma),
\end{aligned}
$$

where

$$
\begin{array}{r}
\phi_{1}(\sigma)=|\sigma|^{\frac{1}{2}} \operatorname{sign}(\sigma)+|\sigma|^{\frac{3}{2}} \operatorname{sign}(\sigma) \\
\phi_{2}(\sigma)=\frac{1}{2} \operatorname{sign}(\sigma)+2 \sigma+\frac{3}{2} \mu^{2}|\sigma|^{2} \operatorname{sign}(\sigma)
\end{array}
$$

and $k_{1}$ and $k_{2}$ are constant positive gains dependent on $L$. The variable $z_{0}$ and $z_{1}$ are the estimations of $\eta_{0}$ and $\dot{\eta}_{0}$, respectively. Indeed, $z_{1}(t)$ converges exactly to $\dot{\eta}_{0}$ in finitetime, with the convergence time independent of the initial differentiation error (see [1] for details).

\section{Extremum Seeking Problem Statement in PEM FCGS}

\section{A. PEM Fuel Cell Generation System}

As stated in the introduction, fuel cells represent a radically different approach to energy conversion, one that could replace conventional power generation technologies in a wide variety of applications, from automotive and stationary power systems to portable appliances. In particular, a proton exchange membrane (PEM) fuel cell is an electrochemical device that converts hydrogen chemical energy into electric power energy, without the intermediate production of mechanical work and with water and heat as only by-products [4], [5]. Nevertheless, improvements in this field of technology require interdisciplinary research and the development of new technologies. From the automation control point of view, the natural step is to face the challenge of designing and implementing reliable control strategies in order to improve the efficiency of the actual fuel cell generation systems, improving its operations ranges and ensuring optimal performance.

With regard to models for FCGS, there are several different approaches that describe the dynamic behaviour of autonomous PEM fuel cell generation systems, but only a few are suitable for control design purposes. Among them, one of the most complete and accurate models available in the open literature was developed by J. Pukrushpan et al. in the Mechanical Department of the Michigan University. This model provides a detailed description of the dynamics of a $75-\mathrm{kW}$-high pressure FC stack fed by a 14-kW air turbo compressor. The system is sized to represent the high pressure FC stack used in the Ford P2000 fuel cell electric vehicle.

\section{B. Nonlinear System Model}

Considering that in the model presented in [6] the anode subsystem is decoupled from the cathode subsystem and does not enter in the air control loop, its dynamics can be neglected and the system order is reduced by one [5]. 
As a result of the reduction and rebuilding work performed on Pukrushpan et al. model, the following sixth order control design model can be proposed [5]:

$$
\begin{gathered}
\dot{x}=F(x, u, t)=f(t, x)+g(t, x, u) \\
x \in \mathbb{R}^{6} ; u \in \mathbb{R} ; f: \mathbb{R}^{6} \rightarrow \mathbb{R}^{6} ; g: \mathbb{R}^{6} \rightarrow \mathbb{R}^{6}
\end{gathered}
$$

with $f$ and $g$ piece-wise continuous on $t$ and sufficiently smooth on $x$ :

$$
x=\left[\begin{array}{llllll}
\omega_{c p} & P_{s m} & m_{s m} & m_{O_{2}, c a} & m_{N_{2}, c a} & P_{r m}
\end{array}\right]^{T}
$$

- $x_{1}=\omega_{c p}$ : angular speed of the compressor motor that feeds the stack cathode through the supply manifold.

- $x_{2}=P_{s m}$ : total pressure inside the supply manifold, consisting of the sum of the partial pressures of the gases that constitute the air (oxygen, nitrogen and water vapour).

- $x_{3}=m_{s m}$ : total mass of air in the supply manifold, consisting of the sum of the instantaneous masses of oxygen, nitrogen and water vapour.

- $x_{4}=m_{O_{2}, c a}$ : instantaneous oxygen mass in the stack's cathode channels. This state is affected by the oxygen consumed in the reaction, the amount of oxygen coming from the supply manifold and the oxygen mass outgoing through the return manifold.

- $x_{5}=m_{N_{2}, c a}$ : instantaneous mass of nitrogen inside the stack's cathode channels. It only relies on the incoming nitrogen from the supply manifold and the outgoing nitrogen that leaves the stack through the return manifold.

- $x_{6}=P_{r m}$ : total pressure inside the return manifold, consisting of the sum of the partial pressures of the gases that constitute the air.

The control input $u(t)$ is the voltage of the compressor DC motor $V_{c p}$.

Suppose now that the control problem of $W_{c p}$ regulation is solved, for instance by the stabilizing Super Twisting controller proposed in [3]. Then, the continuous-time steepest descent algorithm (4) presented in section II, can be tuned to determine the flow reference and find the optimum operating value for each power load $P_{\text {load }}$.

\section{Minimization Problem}

The objective of the case study is to optimize the hydrogen consumption of the FCGS in every operating condition, minimizing the stack current demand under different load conditions. Note that the consumed hydrogen in the reaction $\left(W_{H_{2}, \text { react }}\right)$ is directly related to the stack current $\left(I_{s t}\right)$

$$
W_{H_{2}, \text { react }}=G_{H_{2}} \frac{n I_{s t}}{2 F}
$$

where $G_{H_{2}}$ stands for the molar mass of hydrogen, $n$ is the total number of cells of the stack and $F$ the Faraday's constant [6]. Therefore, the optimization procedure can be stated as a the problem of minimizing the real-valued objective function

$$
y=h(x, u)=I_{s t}=\frac{P_{\text {load }}}{V_{\text {st }}},
$$

subject to the constraint $\dot{x}=0$, where $P_{\text {load }}$ is the power required by an external load and $V_{s t}$ is the fuel cell stack voltage. Further details of the model, assumptions and operating conditions can be found in [6].

The system efficiency optimization can be achieved by regulating the air mass flow entering the stack cathode. Suppose that a proper low level controller ensures the reference comburent flow, then the load demand will be satisfied with minimum fuel consumption if an efficient flow reference manager is designed. In addition, oxygen starvation could be averted in order to extend the stack lifetime. To this end, the following air flow reference is proposed based on (4):

$$
\dot{W}_{c p, r e f}(t)=-\mathbf{1}_{\left[t_{1}, \infty\right)}(t) \cdot k_{u} z(t)+d(t),
$$

where $z(t) \in \mathbb{R}$ is an estimate of $\frac{\partial H}{\partial u}$ at time $t$ and $k_{u}=$ $4 \times 10^{-7}$ is the algorithm gain for the current system. A sinusoidal ditter signal $d(t)$ was applied in order to ensure persistent excitation for the estimator (5).

\section{PEM FCGS Operation Ranges and Minimums of Hydro- gen Consumption}

The steady state map of the analysed system (static relationship between $W_{c p}$ and $I_{s t}$ at different power loads) is depicted in the following figure:

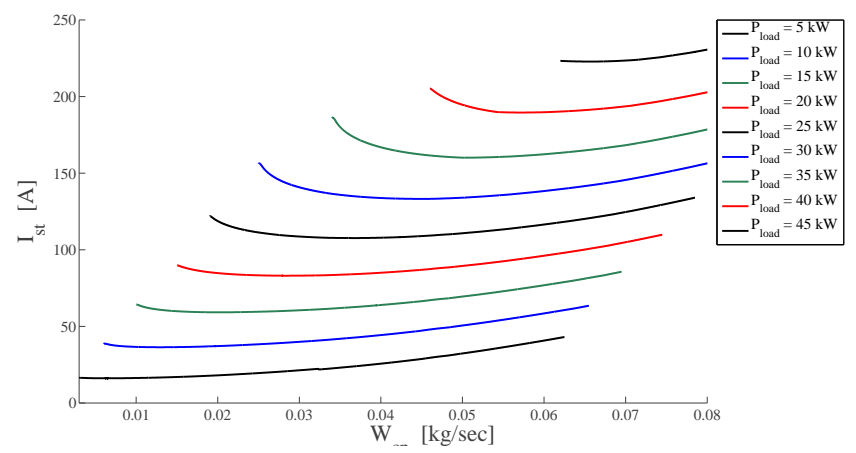

Fig. 3. Steady-state analysis of the system performance in different load conditions

Notice that low air mass flows implies low stack voltage and, hence, higher stack current in order to keep $P_{\text {load }}$ constant. At the same time, a higher air mass flow would require a higher compressor current, increasing $I_{s t}$. Thus, if continuity holds, there must be a minimizing value of air mass between the two extrema of air mass flow.

\section{Simulation Results}

\section{A. Algorithm Performance}

In this section, the FCGS performance is evaluated under the action of the extremum seeking supervisor control (19). Then, the features of the designed algorithm are examined through simulation tests, which aim to demonstrate its nominal tracking performance. To this end, a series of power loads (ranging from $15 \mathrm{~kW}$ to $40 \mathrm{~kW}$ ) were chosen in order to illustrate the air regulation performance in a wide range of operation (Fig. 4).

Note that abrupt and significant changes in the amplitude of the load demand $\left(P_{n e t}\right)$ were considered to test the proficiency of the algorithm under exacting operating conditions. 

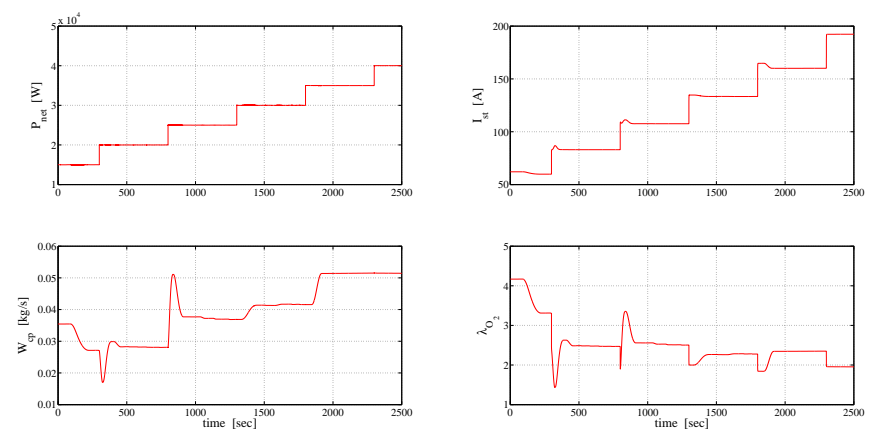

Fig. 4. Extremum seeking algorithm test: system dynamical

In Fig. 4, four different variables can be simultaneously appreciated, the power load $\left(P_{n e t}\right)$, the stack current $\left(I_{s t}\right)$, the compressor air flow $\left(W_{c p}\right)$ and the fuel cell oxygen stoichiometry $\left(\lambda_{O_{2}}\right)$, defined as:

$$
\lambda_{O_{2}}=\frac{W_{O_{2}, \text { ca,in }}}{W_{O_{2}, \text { react }}}
$$

with $W_{O_{2}, c a, i n}$ the oxygen partial flow entering the cathode and $W_{O_{2} \text {,react }}$ the oxygen flow consumed in the reaction.

In Fig. 5 it is shown that the current optimization (and then the oxygen minimization) is successfully achieved for all the tested operating conditions, obtaining efficiency improvements up to $20 \%$. Moreover an adequate comburent flow is always ensured through the stack while the load demand is satisfied with minimum fuel consumption.

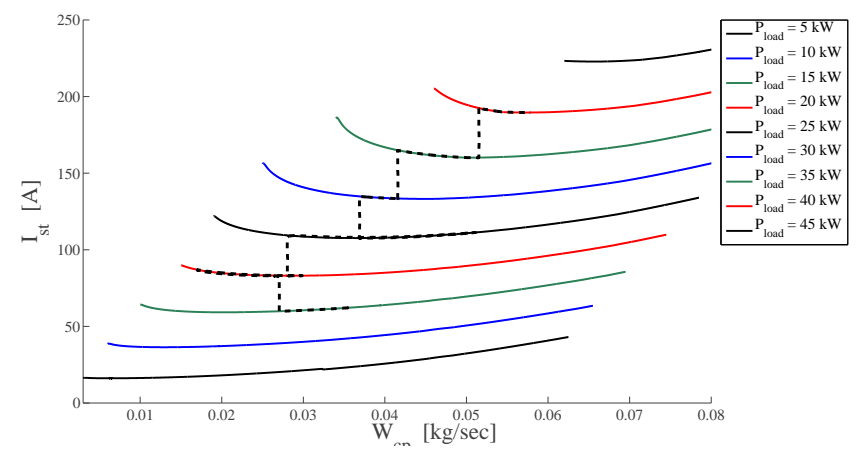

Fig. 5. Extremum seeking algorithm test: system trajectory on the static map

\section{COnClusions}

A general extremum seeking algorithm was presented. A feasibility study of its implementation in PEM fuel cells hydrogen minimization was carried to assess its performance. The algorithm evaluation has been conducted using a benchmark model of a fuel cell system for an electric vehicle. The analysis has established the viability of the presented technique in fuel cells, aiming to improve its energy efficiency.

Taking into account several features, such as the natural time constants of the controlled system, robustness and implementation simplicity, the extremum seeking algorithm is shown to be a highly efficient solution for this challenging problem, proving to be capable of robustly tracking the optimal hydrogen consumption.

Now that the suitability of the approach has been confirmed, the following stage will be the development and implementation of these algorithms in actual fuel cell plants.

\section{REFERENCES}

[1] Cruz-Zavala, E., Moreno, J.A., Fridman, L., 2011. Uniform Robust Exact Differentiator. IEEE Transactions on Automatic Control, Vol. 56 (11), 2727-2733.

[2] EG\&G Technical Services, Inc.(2004) Fuel Cell Handbook (Seventh Edition). U.S. Department of Energy-Office of Fossil Energy.

[3] Kunusch, C., Puleston, P., Mayosky, M. , Riera, J., 2009. Sliding mode strategy for PEM fuel cells stacks breathing control using a SuperTwisting algorithm, IEEE Transactions on Control System Technology 17, 167-174.

[4] Kunusch, C., Puleston, P., Mayosky, M., Husar, A., 2011. ControlOriented Modelling and Experimental Validation of a PEMFC Generation System, IEEE Transactions on Energy Conversion, Vol. 26 (3), 851-861.

[5] Kunusch, C., Puleston, P., Mayosky, M., 2012. Sliding-Mode Control of PEM Fuel Cells. Series of Advances in Industrial Control, Springer. ISBN 978-1-4471-2430-6.

[6] Pukrushpan, J., Stefanopoulou, A., Peng, H., 2004. Control of Fuel Cell Power Systems. Springer UK.

[7] Moreno, J., 2011. Lyapunov Approach for Analysis and Design of Second Order Sliding Mode Algorithms. Springer. Chapter 4, 122150.

[8] Utkin, V., Gulder, J., Shi, J. 1999. Sliding mode Control in Electro Mechanical Systems, Taylor and Francis, London.

[9] Hartman, P. 2002, Ordinary Differential Equations, Society for Industrial and Applied Mathematics, Philadelphia.

[10] Khalil, H., 1996, Nonlinear Systems, Prentice-Hall, New Jersey. 